\title{
Impact of Endodontically Treated Teeth on Systemic Diseases
}

\section{Johann Lechner ${ }^{1 *}$ and Volker von Baehr ${ }^{2}$}

${ }^{1}$ Department of Integrative Dentistry, Grünwalder Str. 10A, 81547, München, Germany

${ }^{2}$ Department of Immunology and Allergology at the Institute for Medical Diagnostics in MVZ GbR, Nicolaistr 22, 12247, Berlin, Germany

\begin{abstract}
Background: This study compares the radiographic distribution of apical periodontitis (AP) in rootfilled and endodontically treated teeth among healthy controls and patients with systemic diseases; the incidence of AP was almost twice as high in the latter group.

Objective: The question arises as to whether the biogenic amines (mercaptan/thioether/hydrogen sulfide) originating from endodontically treated teeth have systemic, subtoxic and immunological effects. Method: In order to determine this, local hydrogen sulfide measurements of endodontically treated teeth were combined with the laboratory serum analyses of modified proteins to assess the relationship of these compounds with type IV immune reactions.
\end{abstract}

Results: It was found that $42.5 \%$ of the group with systemic diseases showed immunological disturbance as a result of root-filled teeth. Furthermore, the presence of AP was almost three times higher than in the control group ( $17.2 \%$ versus $5.9 \%$, respectively).

Conclusion: In summary, the data demonstrates that local pathologies caused by endodontically treated teeth may increase immunological and systemic dysfunction.

Keywords: Endodontic treatment; Root filling; Mercaptan/ Thioethers; Systemic diseases

Abbreviations: AP: Apical Periodontitis; HC: Healthy Control; IFN-Г: Interferon Gamma; IL-10: Interleukin-10; Rft: Root-Filled Tooth/Teeth; Syd: Systemic Disease; Vhc: Volatile Hydrogen Compounds; Vshci: Volatile Sulfur Hydrogen Compounds Indicator

\section{Background}

Dentists have learned to treat inflamed and necrotic teeth with root fillings and various endodontic procedures have been developed for this purpose. Those in support of root canal therapy generally hold the view that such procedures are safe and successful, provided that the patient has no complaints concerning pain or problems with their bite. This view is further supported when radiographs show no sign of an inflammatory reaction. Those in favour of root canal treatment contend that with modern treatment methods any systemic immunological effects of root-filled teeth (RfT) can be avoided. However, inflammation only becomes apparent in X-ray images when associated with bone and tissue degradation. The available scientific evidence also indicates that diabetes is significantly associated with a higher prevalence of periapical radiolucencies in endodontically treated teeth [1]. Conversely, critics of root canals hold the view that they may contribute to immunologically based diseases and consider X-ray imaging to be insufficient to determine the possible systemic effects of toxins derived from root fillings. They contend that bacteria and other pathogens can survive in endodontically treated teeth and their metabolic products - mercaptan and thioethers - can produce immunological and subtoxic effects.

AP is a chronic inflammatory disorder of the periradicular tissues caused by bacterial invasion at the apex of the tooth root [2]. Evidence from epidemiologic studies suggests that the association between AP and various diseases is not new as shown in numerous publications [3]. For example, apical infections like AP are associated with increased rates of myocardial infarction, with acute coronary syndromes occurring 2.7 times more frequently in those patients with such infections [3], as well as clinical depression, increased severity of depression and a reduced quality of life [4]. Increased translocation of gram-negative bacteria may also be associated with AP [5,6]. AP causes not only local inflammatory tissue destruction but also systemic inflammatory responses which may ultimately predispose an individual to systemic disease, including cardiovascular disorders [7-9]. A study on a total of 248 patients after acute myocardial infarction and 249 healthy controls underline that patients, who have experienced a myocardial infarction, had more missing teeth and a higher number of inflammatory processes, especially of endodontic origin, than healthy patients [10]. Subjects presenting lesions of endodontic origin or pulpal inflammation had an increased risk of developing coronary heart disease. Chronic oral diseases may be an unconventional risk factor for coronary heart disease [11]. The value for the total amount of bacterial DNA in in the coronary plaque was 16 times higher than that found in their blood samples. Bacterial DNA typical for endodontic infection, mainly oral viridans streptococci, was measured in $78.2 \%$ of thrombi, and periodontal pathogens were measured in $34.7 \%$. Dental infection and oral bacteria, especially viridans streptococci, may be associated with the development of acute coronary thrombosis [12]. There is also a significant association between periodontitis and depression [13]. However, there is no data as to whether sulfur hydrogen levels in the root canals of patients with AP may be associated with systemic and immunological diseases. The study presented herein is one of the first to statistically link a patient group with multiple systemic and immunological diseases with endotoxin levels originating from AP. This study showed that there were significantly increased root canal endotoxin levels in patients with AP in comparison with healthy controls

*Corresponding author: Johann Lechner, Head of Clinic Integrative Dentistry Munich Gruenwalder Str. 10A, 81547 Muenchen, Germany, Tel: 0049-896970129; Fax: +49-89-6925830; E-mail: drlechner@aol.com

Received March 05, 2018; Accepted March 23, 2018; Published March 31, 2018

Citation: Lechner J, Baehr Vv (2018) Impact of Endodontically Treated Teeth on Systemic Diseases. Dentistry 8: 476. doi:10.4172/2161-1122.1000476

Copyright: $\odot 2018$ Lechner J, et al. This is an open-access article distributed under the terms of the Creative Commons Attribution License, which permits unrestricted use, distribution, and reproduction in any medium, provided the original author and source are credited. 
(HCs) without periodontitis. The link between AP and increased root canal endotoxin levels may explain previous findings that inflammation in periodontal disease is mediated by macrophage proinflammatory cytokines [14,15]. Increased endotoxin levels activate the toll-like receptor TLR $2 / 4$, thereby increasing inflammatory responses leading to macrophage activation [16].

\section{Study Objective}

The aim of the present study was to determine the extent to which X-ray imaging may be used to identify conspicuous and inflammatory reactions in the case of AP in a group of 98 patients with chronic systemic immunological disorders. The results were compared with a second patient group recruited from a university research subject pool; these individuals showed no evidence of any comparable systemic diseases [3].

\section{Materials and Methods}

\section{Patient cohort with systemic diseases}

This study is a retrospective case-control study that obtained approval waiver by the accredited IMD-Berlin Forensic Institute (DIN EN 15189/DIN EN 17025). All patients provided their written informed consent. The following factors were identified for each patient: age, sex and number of remaining teeth, (excluding the third molars). The sample population in our retrospective cross-sectional study consisted of 98 adult patients $(\mathrm{m}=45 ; \mathrm{f}=53$; average age (years): 54 ; range (years): 27-75) with a total of 1,783 teeth. On average, each patient in our sample had 18.2 teeth.

All patients underwent medically necessary radiographic examinations of their teeth. The inclusion criterion was the presence of a systemic disease (SyD). Those patients with such a disease were subject to an investigation for AP associated inflammation using threedimensional (3D) digital volume tomography (DVT) respectively cone beam computed tomography (CBCT). All patients were referred to our practice by treating specialists. The patient group with SyD comprised the following: seven patients with various tumor-related diseases, (two with prostate cancer, two with colorectal cancer and three with breast cancer); 32 patients with chronic fatigue syndrome and systemic immunological exhaustion; 19 patients with rheumatoid complaints; nine patients with degenerative neurological diseases, (four with multiple sclerosis, three with amyotrophic lateral sclerosis and two with Parkinson's disease); 18 patients with atypical facial pain and trigeminal neuralgia; and 12 patients with various intestinal symptoms which may indicate systemic disorders or diseases as yet undiagnosed. The predominant exclusion criteria was bleeding at the gingival margin and/or inflammation of the periodontal socket for two reasons: (i) paper pins used to test for Thio/Merc outgas would be colored red and thus falsify the toxin measurement of the volatile sulfur hydrogen compounds indicator (vSHCI); and (ii) any remaining anaerobic bacteria in the socket may also produce vSHC and thereby falsify the measurement of outgas from root canals.

\section{Investigations with CBTC/DVT}

For each tooth the presence of AP and the quality of each root filling were assessed using 3D-DVT/CBCT. This medical imaging technique was selected, rather than two-dimensional orthopantomography (2D-OPG), since a large number of scientific publications examining the problems associated with $\mathrm{X}$-ray diagnostics show that periapical radiographs - the most commonly used method are unsuitable for assessing the success of endodontic treatment. The latter imaging technique is only two dimensional and thus may not provide the necessary information on the orthogonal planes [17]. There are several well-known limitations of 2D-OPG which have been scientifically proven: apical changes cannot be reliably assessed with $2 \mathrm{D}-\mathrm{OPG}$ and $34 \%$ of Aps are not identified [18]. Furthermore, endodontists were found to fail to see at least one root canal in $40 \%$ of molar teeth. Thus, one-third to one-half of all 2D-OPG scans are insufficient for dental diagnostics [19]. In dental practice the structure of the jawbone is generally assessed using 2D-OPG, particularly in instances of cortical bone damage. However, as previously shown, there are considerable limitations in the assessment of cancellous bone which must be considered [19-21]. Despite the recent advances in digital X-ray technology and improved imaging modalities, the limitations of imaging cancellous bone persist [22].

Figure 1 demonstrates the critical importance of using both 2D-OPG and 3D-DVT/CBCT to investigate endodontically treated teeth and make a complete diagnosis. Although AP is clearly visible at the mesiobucal root using 3D-DVT/CBCT, the 2D-OPG image is inconspicuous. Following removal of the crown, the dentine was clearly markedly colored by biogenic amines (right panel) which diffuse into the periodontal socket of the tooth and can be made visible using a volatile hydrogen sulfide indicator.

\section{DVT Investigation for systemic diseases}

Each patient in our study was X-rayed between February 1, 2016 and June 30, 2016 using 2D-OPG and 3D-DVT/ CBCT; only the CBCT scans were used in this evaluation. CBCT scans were recorded using the KODAK 9000 Extraoral Imaging System X-ray machine (KODAK, Rochester, NY, USA). Scans were evaluated in a darkened room using the KODAK Dental Imaging Software $6 \cdot 12.11 .0^{\circ}$ on a 30 -inch monitor with a resolution of 2560 x 1600 pixels (Dell 3008WFP; Dell Inc., Round Rock, TX, USA). Our evaluation criteria were based on those used in a comparative study on root fillings [3]. For AP, radiolucency was defined for the apical part of the root; the apical part was more than twice the width of the lateral part of the periodontal ligament De Moore et al. [3]. The patients were not screened for pre-existing periapical pathology before the roots were treated. In the case of multi-root teeth, the root was classified according to the root with the most severe AP. Endodontically treated or root-filled teeth (RfT) were presumed to contain a radiopaque material in the root canals. An root filling was considered to be adequate when it was contained within the tooth, it was not more than $2 \mathrm{~mm}$ from the radiographic root tip and no visible cavities were present. A root filling that was more than $2 \mathrm{~mm}$ from the radiographic tip containing cavities or where the apical foramen was

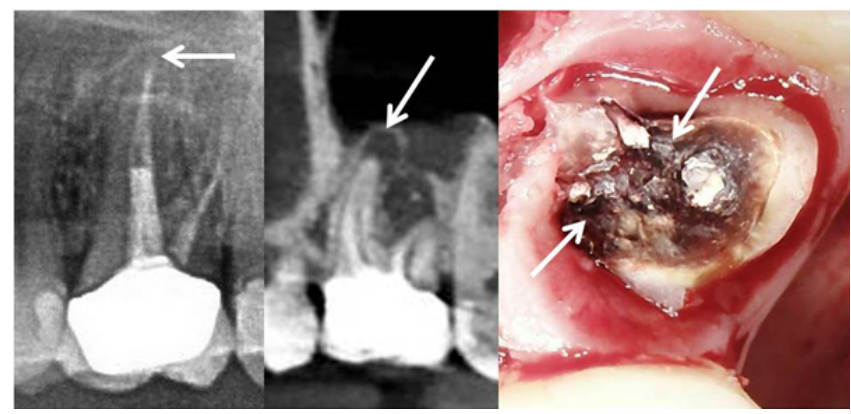

Figure 1: Comparison of two techniques: 2D-OPG and 3D-DVT/CBCT. 3D-DVT/CBCT clearly shows AP (centre panel) when compared to the 2D-OPG (left panel). Right panel shows dentine blackening caused by biogenic amines after removal of the crown. 
overfilled was considered inadequate. In the case of multiple-root teeth, the root was categorized according to the most insufficient root filling (Loftus et al., 2005, Siqueira et al., 2005, Tavares et al., 2009, Kalender et al. 2013, Di Filippo et al., 2014) from [3].

\section{Semiquantitative determination of volatile hydrogen sulfides in tooth sockets}

The problem associated with the biogenic degradation of necrotized pulp has been extensively investigated and discussed in dentistry. Biogenic amines - like mercaptan, thioether, hydrogen sulfide, all of which are volatile hydrogen compounds ( $\mathrm{vHC}$ ) - have chronic subtoxic effects and their effects upon the immune system may be evident in endodontically treated teeth. A control X-ray image is typically standard practice and the only method used for the diagnostic assessment of RfT. However, X-ray scans are insufficient since chemically defined toxins cannot be visually identified $[23,24]$. We thus expanded our investigation to incorporate an additional evaluation criterion in order to semiquantitatively determine the presence of volatile hydrogen sulfide compounds [2]. With this chairside test, hydrogen sulfide can be displayed using a volatile sulfur hydrogen compounds indicator (vSHCI). The procedure is painless and simple to administer: a paper tip is inserted into the sulcus of the suspicious tooth and removed after one minute, whereupon it is inserted into the volatile compound reagent container. After 5 minutes, the staining of the reagent is read: the more hydrogen sulfide compounds present in the sample, the more the indicator liquid turns yellow. The scale of yellow intensity ranges from 0 (no load) to 5 (very strong load). The degree of coloration of the reagent may be used to semiquantitatively determine the amount of toxin that can be resorbed in the sulcus (Figure 2).

\section{Determination of immunological sensitization by biogenic amines}

To establish a possible relationship between conspicuous X-ray findings on DVT and the presence of disease, a blood test to assess immune system sensitization by biogenic amines was carried out in 73 patients. As was the case in a previously published study [24], the study investigated whether patients' blood cells secreted interferon gamma (IFN- $\gamma$ ) or interleukin (IL)-10 in vitro following contacts with biogenic amines, thereby establishing immunological sensitization. Lymphocytes and monocytes were isolated from the patients' blood by density gradient centrifugation. Thereafter, $1.2 \times 106$ cells were transferred and the monocytes primed to antigen-presenting cells with granulocytemacrophage colony-stimulating factor (GM-CSF). Twenty-four hours after having added mercaptans/thioethers to the patients' cells, IFN- $\gamma$ and IL-10 were analyzed in the cell culture supernatant.

\section{Statistics}

Statistical analysis was performed using IBM SPSS, version 19 (IBM Corporation, Armonk, NY, USA). All data was presented as a mean \pm standard mean error. Data was considered significant where the value was $<0.05$.

\section{Results}

CBCT findings in the group of patients with systemic diseases. In 98 patients with $\mathrm{SyD}$, a total of 1,783 teeth underwent CBCT. Of these, 324 teeth were endodontically treated (18.10\%), while 323 of the endodontically treated teeth showed signs of AP (95.10\%) (Figure 3

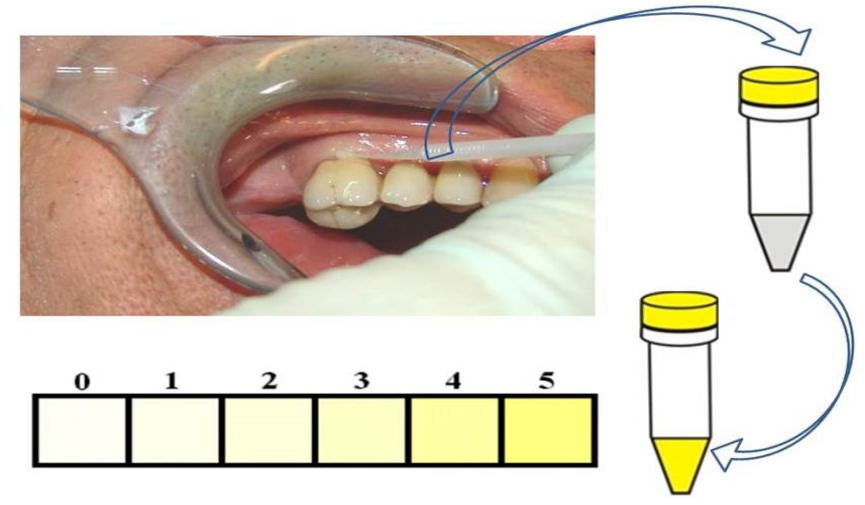

Figure 2: Semiquantitative determination of toxin intensity resorbed by the periodontal socket.

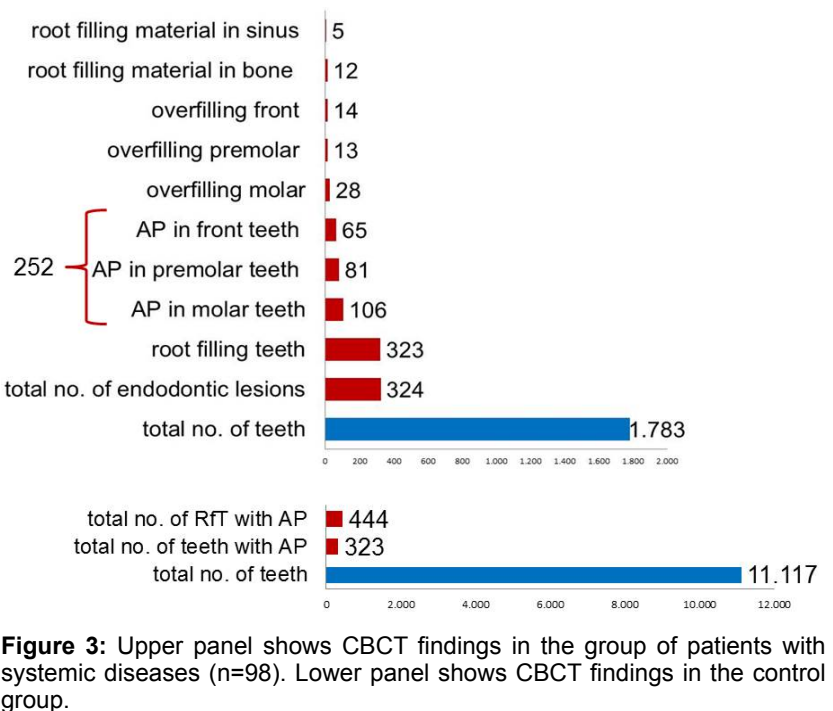
group.

upper panel). Lower panel shows CBCT findings in the control group.

\section{$\mathrm{X}$-ray findings in the control groups}

At the Leuven University Dental Clinic, 804 patients received a CBCT scan between January 1, 2013 and January 1, 2014. This cohort served as the control group. Control group was chosen from university research because of diagnostic and statistical reliability. The cited study was conducted in a general dental clinic and no SyD were documented for the cohort in which 631 scans of permanent dentition were evaluated with a total of 11.117 teeth [3]. The number of teeth in which AP was present was 656 and the number of RfT with AP was 444 .

\section{Intensity of the local excretion of biogenic amines in periodontal sockets}

Toxin intensity was measured in $212 \mathrm{RfT}$ of the SyD patient group seven patients with different tumors, 32 with chronic fatigue syndrome, 19 with rheumatoid complaints, nine with neurological degeneration, 18 with facial pain and trigeminal neuralgia, and 12 with various intestinal symptoms using a vSHCI; the cumulative average intensity was 681 ( \pm 3.3 STDEV). This value corresponds to a grading based on 
the 6-point scale employed in this study where scores ranged from 0 to 5 in ascending order of concentration; accordingly, a local toxin release of 3.21 was observed per RfT.

\section{Sensitization of the immune system to biogenic toxins originating from endodontically treated teeth}

In 73 of the 98 patients with SyD, an investigation was carried out to determine immunosensitization to tooth toxins. In 13 patients of the SyD cohort $(\mathrm{m}=7 ; \mathrm{f}=6)$, a TH1-dominant cellular sensitization, which was identifiable due to elevated IFN- $\gamma$ values, was observed. These patients had an average IFN $-\gamma$ value of $39.9 \mathrm{IU} / \mathrm{mL} \pm 4.17$ STDEV (normal value: $<0.10$ ). An additional 18 patients of the SyD cohort $(\mathrm{m}=7 ; \mathrm{f}=11)$ showed IL-10 induction which also indicated sensitization to tooth toxins. The mean value for IL-10 was $93.7 \mathrm{pg} / \mathrm{mL} \pm 72.49$ STDEV (normal value: $<10.0$ ). In summary, 31 of 73 patients $(42.5 \%)$ showed signs of immunosensitization to $\mathrm{vHC}$ in association with the presence of RfT.

\section{Discussion}

The complexity and breadth of the possible causes of SyD require advanced scientific inquiry and investigation. Etiological factors are involved in system integration, such as polymorphisms, epigenetic factors, functional modulations, environmental influences and immunological engrams of the neuro-endocrine and immune systems [25] which are initially triggered and then repeatedly and uniformly released, even in the absence of the primary trigger. It is likely that advanced stages of chronic toxic stress disrupts normal homeostatic processes and this becomes progressively more difficult to reverse [26].

The relationship between endodontic measures and AP are presented and discussed in numerous publications [27-30]. The findings of four relevant studies may be summarized as follows: i) the prevalence of periradicular disorders in RfT remains high in Scottish populations [27]; ii) teeth with AP appear more frequently in patients with RfT than in those without, $(39 \%$ versus $9 \%$, respectively; $\mathrm{P}<0.001)$, and $25 \%$ of RfT displayed AP [28]; iii) the technical quality of root fillings in an adult Irish population was poor and consistent with the high prevalence of AP [29]; iv) there was a high incidence of AP in RfT - the total number of RfT was 93, and of these, 60 (64.5\%) had AP [30].

To address the objective of this study, we employed a direct comparative study using as a healthy control group ( $\mathrm{HC}$ ) the cohort of 804 patients described in a study conducted in Belgium [3]. Using CBCT scans, the cohort was established as having RfT with AP: 631 CBCT scans of 11,117 permanent teeth were evaluated at the University Clinic of Leuven between January 1, 2013 and January 1, 2014 and no SyD were documented. A total of 656 teeth showed signs of AP, while AP was detected in 444 of RfT.

Data collection was carried out in both study series using DVT scans. In our comparison, the endodontic procedures in both groups were carried out according to the same European good clinical practice standards. Although more than six-fold teeth were examined in the control group (11.117/1.783), the number of cases of AP in the control group was only twice as large as that of the SyD group (656/307); moreover, the number of RfT in which AP was present was closely matched (444/307) (Figure 4a). When comparing the HC and SyD groups of those teeth examined, a ratio of $6: 1$ was found for RfT in the HC group; conversely, in the SyD group, the ratio of RfT was 4:1. Taking the number of X-rayed teeth of both groups as $100 \%$, the following comparative results were found (Figure $4 \mathrm{~b}$ ):

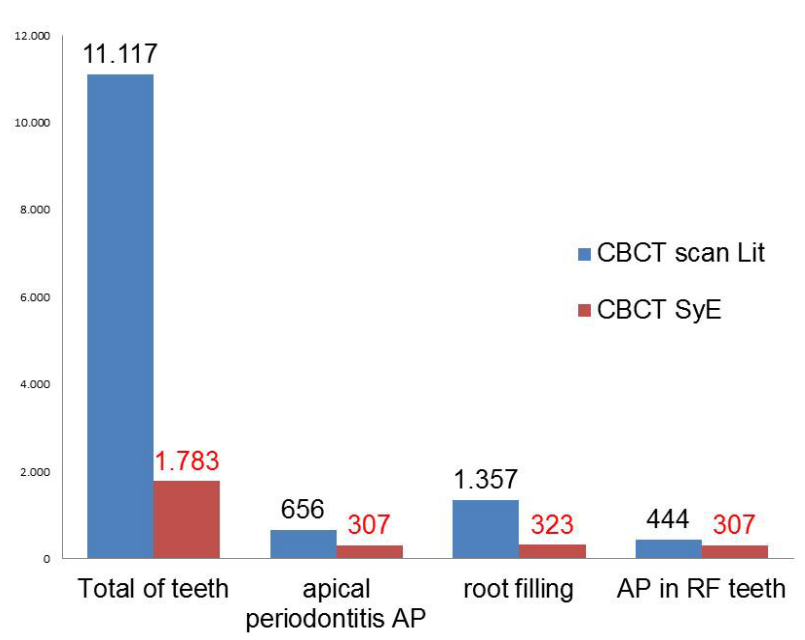

Figure 4a: Comparison of CBCT findings in total numbers of teeth in patients with systemic diseases $(n=1.783$ in red columns) and healthy controls $(n=$ 11.117 in blue columns)

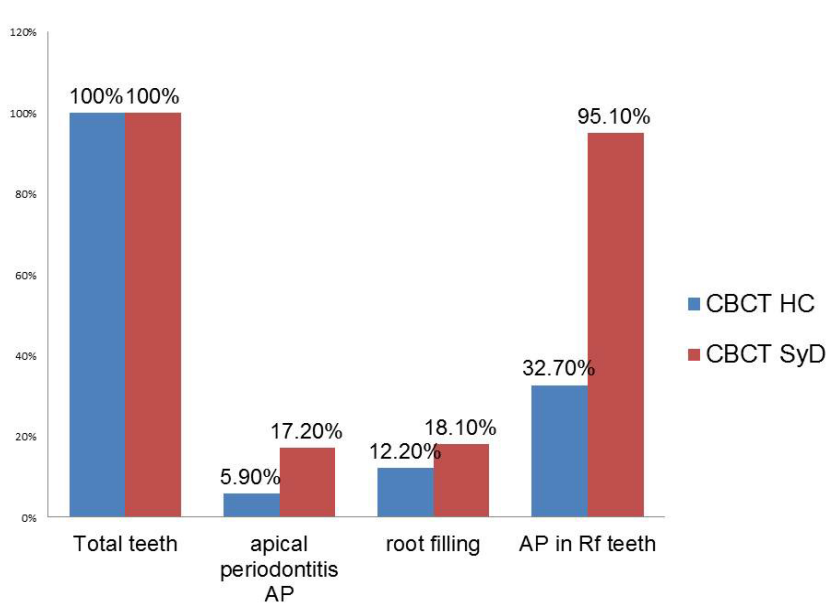

Figure 4b: Comparison of CBCT findings in teeth $(n=11.117)$ of healthy controls (blue columns) versus teeth of patients with systemic diseases ( $n=$ 1.783 ) as expressed in percentages.

Overall, the SyD group demonstrated an almost three times greater incidence of AP than the HC group (17.2\% / 5.9\%); AP was evident in 95.1\% of RfT in the SyD group, while a rate of only $32.7 \%$ was observed in the HC group indicating a triple-fold increase of AP in those patients with SyD. The number of RfT in patients with SyD was only slightly higher than that of the HC group (18.1\% / 12.2\%).

In the group of patients with $\mathrm{SyD}$, the prevalence of $\mathrm{AP}$ was higher than in other epidemiological studies [27-30]. At the same time, the number of RfT showing signs of AP in patients with SyD was three times greater than in the healthy control group.

\section{Discussion of the semiquantitative determination of volatile hydrogen sulfide in periodontal sockets}

The field of modern endodontics is aware of the problem of bacterial colonization in the dentine tubules and new procedures are constantly being developed to minimize this problem and any possible associated risks. To date, scientific methods for detecting bacterially produced 
toxins have been lacking. Their detection by toxin measurements provide a well-supported answer to the question of whether a RfT in those patients with SyD should be removed or whether it should be revised and refilled, even in the absence of radiographic changes. It is known from numerous publications [31-33] that biogenic amines (mercaptan/thioether/hydrogen sulfide) may emerge from RfT thereby triggering chronic subtoxic and, in turn, systemic and immunological effects. Some of the most well-known studies have presented the comparison between radiographic and histological findings at the root tips of RfT postmortem. For instance, of 19 recorded findings, five (26\%) appeared to be radiographically inconspicuous but, nevertheless, showed histological signs of inflammation [34]. The authors of the study state that "these areas often contained bacteria and inflamed or necrotic tissue, although the root canal filling was radiographically impeccable. Moreover, not all periradicular inflammations are radiologically diagnosable and a high percentage are asymptomatic" [35]. The results from additional studies confirmed that AP was present in at least half of the samples; in some cases, inflammation in the periapical tissue area was detected only 30 months after the initial procedure. The findings of these studies are consistent with those of Brynolf (Odontol. Rev., 1967) which reported that only $7 \%$ of endodontically treated teeth healed completely. In eight of the 14 samples (57\%), filling material was found beyond the apex. These overfillings regularly caused an inflammatory response [36]. Since anaerobic bacteria from AP produce the cleavage product of L-methionine using 2-ketobutyrate as a source of energy [24], they also produce various types of endo- and exotoxins that act as exogenous pyrogens or cause intoxication in the body. These toxins include thioether/mercaptan from the tooth cavity in each RfT. It is important to note that a vSHCI may also be used to detect systemic immunological relationships between volatile reagents and AP [24]. The results that were obtained after using the vSHCI were documented per tooth and rated on an intensity grading scale ranging from $0-5$. This method was employed for 212 teeth of patients from the 97 patients in our study. In "Bacterial invasion in the dentinal tubules of human vital and nonvital teeth", Nagaoka et al. [36] reported that in vital teeth with fillings that were exposed for more than 150 days, $1.1 \%$ of the dentin tubules were infected with bacteria, whereas in the case of nonvital RfT, $39.0 \%$ of the tubules were infected with bacteria.

\section{The systemic immunological role of thioether compounds}

Since our investigations show an immunological sensitization to RfT in $42.5 \%$ of patients with SyD, it may be concluded that a type IV immune response to modified proteins may be involved in the pathogenesis of such diseases. In sensitized patients, inflammatory cells - primarily lymphocytes - are activated which can cause both local and systemic reactions and the degradation products of biogenic amines may serve as an effective trigger in those patients which test positively for SyD. Figure 5 shows two examples of laboratory findings that were obtained from the group of patients with SyD. A positive result indicates not only that a load was, or is, present but also that mercaptane/ thioether-specific TH1 lymphocytes are present in the peripheral blood which can cause inflammation triggered by mercaptane/thioether IFN- $\gamma$ stimulation (upper panel). Positive IL-10 results are indicative of the mercaptane/thioether-induced activation of immunosuppressive cells, (inhibited immune defense). In Figure 5, the first patient was diagnosed with rheumatoid arthritis, while the second was diagnosed with breast

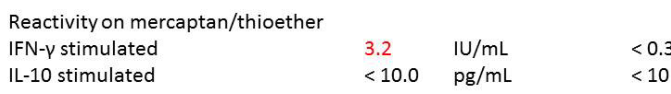

Interpretation:

The TH1-dominant cytokine response to the protein degradation products metacarpan and thioether. Thus, this finding may indicate the presence of a local or systemic inflammatory reaction.

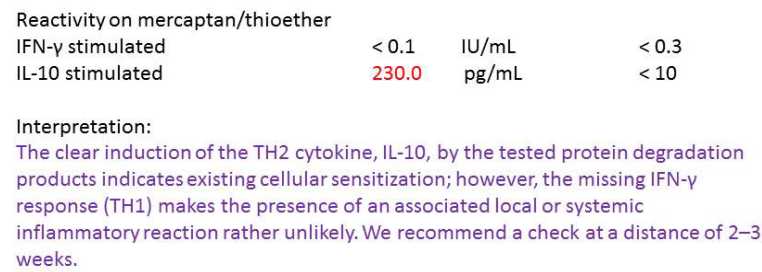

Figure 5: Two examples of laboratory findings in two patients diagnosed with rheumatoid arthritis and breast cancer, respectively.

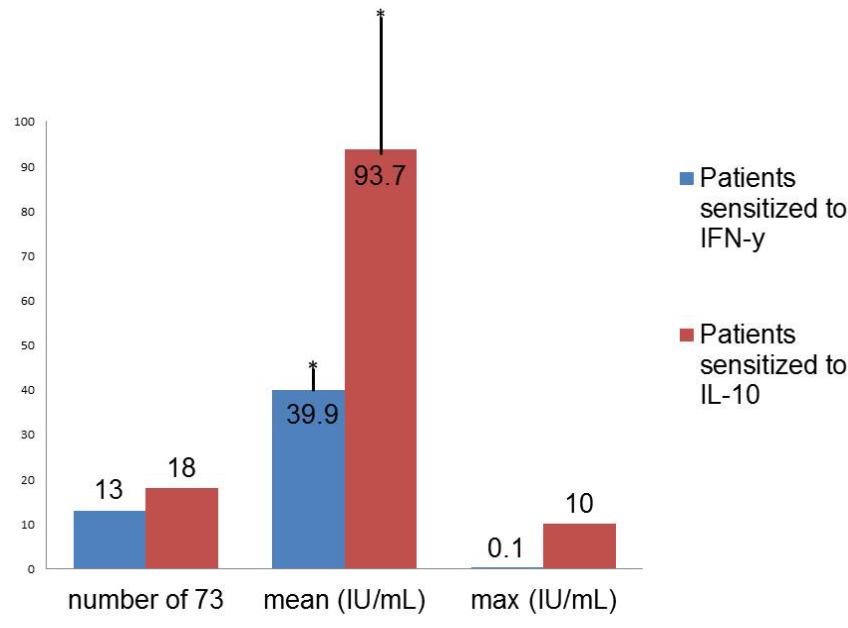

Figure 6: Immunosensitization to $\mathrm{vHC}$ in 73 patients of the SyD cohor separated in IFNy and in IL-10 stimulation.

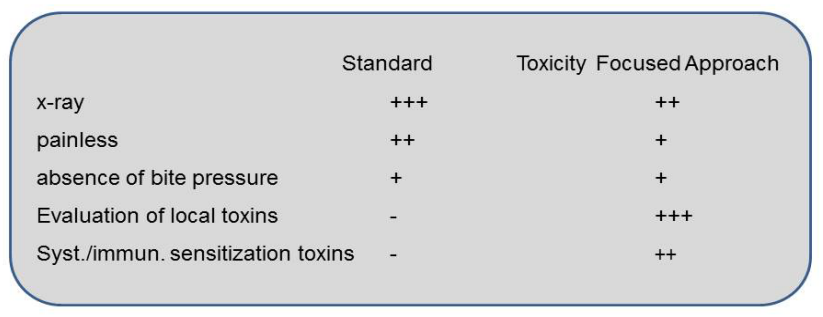

Grading of evaluation criteria for successful endodontical treatment +++ most important; ++ important; + less important; - non-existent

Figure 7: Evaluation criteria to determine the success of endodontic treatment according to standard practice versus a local and systemic toxicity focused approach. 
cancer. Figure 6 shows the immunosensitization to $\mathrm{vHC}$ in 73 patients of the SyD cohort separated in IFN- $\gamma$ and in IL-10 stimulation.

\section{Overview of clinical and diagnostic tools}

The panel in Figure 7 compares the different evaluation criteria for successful endodontic treatment in standard dentistry and presents a further developed systemic approach. While the measurement of local vHC on RfT with a vSHCI in standard dental practice for the "successful evaluation" of endodontic treatment is uncommon, the link of such vHC to SyD may be established readily with an immunosensitization test performed by a specialised laboratory.

\section{Conclusion}

The present study is among the first to focus on the relationship between radiographically visible pathologies in the dental or jawbone region and the presence of SyD. The data presented here do not prove a cause and effect relation. It could be that the patients demonstrated increased AP due the presence of systemic conditions; an interaction of the systemic conditions vice versa cannot be excluded. Notwithstanding the evidence, the consensus is APs are chronic inflammatory processes and triggering conditions in advance of occurring immune diseases. The comparison made between the HC and SyD groups provides the first indication of the possible connections between RfT and SyD, and indicates that endodontically treated and RfT: (i) may enhance immunological and systemic disturbances, and (ii) may be involved in the development of SyD or vice versa, that is, the presence of SyD may influence in some way local inflammatory reactions such as AP. High local $\mathrm{H} 2 \mathrm{~S}$ values with the reagent as well as a high frequency of immunosensitization to biogenic amines in patients with SyD amplify this relationship. In view of the increasing prevalence of immune system diseases, widespread endodontic measures used in dentistry should either be assessed more critically or classified as "successful/ unsuccessful" with reference to additional measurement methods. For practitioners, the semiquantitative, local measurement of volatile compounds, (such as hydrogen sulfide) ${ }^{\star} 1$, as well as systemic studies on the sensitization of the immune system by biogenic amines (thioether/ mercaptans) via increased IL-10 and IFN- $\gamma$ are proposed. More broadly, practitioners performing endodontic procedures should be aware of the relationship between the outcome of endodontic treatment and systemic diseases.

\section{Conflict of Interest}

There is no conflict of interest.

\section{Acknowledgments}

English-language translation and editing of this manuscript were provided by Ilaria Floris. Additional editing was provided by Natasha Gabriel.

\section{References}

1. Segura-Egea Jj, Martín-González J, Castellanos-Cosano L (2015) Endodontic medicine: connections between apical periodontitis and systemic diseases. Int Endod J 48: 933-951.

2. Nair PNR (2006) On the causes of persistent apical periodontitis: a review. Int Endod J 39: 249-281

3. Van der Veken D, Curvers F, Fieuws S, Lambrechts P (2016) Prevalence of apical periodontitis and root filled teeth in a Belgian subpopulation found on CВCT images. International Endodontic Journal 50: 317-329.

4. Gomes C, Martinho FC, Barbosa DS, Antunes LS, Póvoa HCC, et al. (2017) Increased Root Canal Endotoxin Levels are Associated with Chronic Apical Periodontitis, Increased Oxidative and Nitrosative Stress, Major Depression, Severity of Depression, and a Lowered Quality of Life. Molecular Neurobiology Springer Nature 55: 2814-27.
5. Berk M, Williams LJ, Jacka FN, O'Neil A, Pasco JA, et al. (2013) So depression is an inflammatory disease, but where does the inflam- mation come from? BMC Med 11: 1-16.

6. Herath TD, Darveau RP, Seneviratne CJ, Wang CY, Wang Y, et al. (2016) Heterogeneous Porphyromonas gingivalis LPS modulates immunoinflammatory response, antioxidant defense and cytoskeletal dynamics in human gingival fibroblasts. Scientific Reports 6: 1-15.

7. Socransky SS, Haffajee AD (2000) Periodontal microbial ecology. Periodontol 38: $135-187$

8. Amar S, Han X (2003) The impact of periodontal infection on systemic diseases. Med Sci Monit 9: 291-299.

9. Kinane DF, Lowe G (2000) How periodontal disease may contribute to cardiovascular disease. Periodontol 23: 121-126.

10. Willershausen I, Weyer V, Peter M, Weichert C, Kasaj A, et al. (2014) Association between chronic periodontal and apical inflammation and acute myocardial infarction. Odontology 102: 297-302.

11. Pasqualini D, Bergandi L, Palumbo L, Borraccino A, Dambra V, et al. (2012) Association among Oral Health, Apical Periodontitis, CD14 Polymorphisms, and Coronary Heart Disease in Middle-aged Adults. Journal of Endodontics. Elsevier BV 38: 1570-1577.

12. Pessi T, Karhunen V, Karjalainen PP, Ylitalo A, Airaksinen JK, et al. (2013) Bacterial Signatures in Thrombus Aspirates of Patients With Myocardial Infarction. Circulation Ovid Technologies (Wolters Kluwer Health) 127: 12191228.

13. Kumar A, Kardkal A, Debnath S, Lakshminarayan J (2015) Association of periodontal health indicators and major depressive disorder in hospital outpatients. J Indian Soc Periodontol 19: 507-511.

14. Hasturk H, Kantarci A, Van Dyke TE (2012) Oral inflammatory diseases and systemic inflammation: role of the macrophage. Front Immunol 3: 118-138

15. Ara T, Kurata K, Hirai K, Uchihashi T, Uematsu T, et al. (2009) Human gingiva fibroblasts are critical in sustaining inflammation in periodontal disease. $J$ Periodont Res 44: 21-27

16. Lucas K, Maes M (2013) Role of the toll like receptor (TLR) radical cycle in chronic inflammation: possible treatments targeting the TLR4 pathway. Mol Neurobiol 48: 190-204.

17. Liljestrand JM, Mäntylä P, Paju S, Buhlin K, Kopra KAE, et al. (2016) Association of Endodontic Lesions with Coronary Artery Disease. Journal of Dental Research SAGE Publications 96: 234-234.

18. Patel S, Dawood A, Mannocci F, Wilson R, Pitt Ford (2009) Detection of periapical bone defects in human jaws using cone beam computed tomography and intraoral radiography. Int Endod J 42: 507-515.

19. Bumann A. Der aktuelle Stand bildgebender Verfahren, Wissenschaft und Fortbildung BZB November 0959.

20. Schwartz SF, Foster JK (1971) Roentgenographic interpretation of experimentally produced bony lesions. I. Oral Surg 32: 606-612.

21. Ardran CM (1951) Bone destruction not demonstrable by radiograph. $\mathrm{Br} J$ Radiol 24:107.

22. Bender IB, Seltzer S (1961) Roentgenographic and direct observation of experimental lesions in bone. I and II. JADA 62: 152-160.

23. Birchwood G (1996) Osteoporotic bone. Understanding osteoporosis and its treatment: a guide for physicians and their patients. New York: The Parthenon Publishing Group 8-11.

24. Lechner J, von Baehr V (2015) Stimulation of proinflammatory cytokines by volatile sulfur compounds in endodontically treated teeth. Int J Gen Med 9 : 109-118.

25. Kirkpatrick CJ, Fuchs S, Peters K, Brochhausen C, Hermanns MI, et al (2006) Visions for regenerative medicine: interface between scientific fact and science fiction. Artificial Organs 30: 822-827.

26. Godin PJ, Buchman TG (1996) Uncoupling of biological oscillators: a complementary hypothesis concerning the pathogenesis of multiple organ dysfunction syndrome. Crit Care Med 24: 1107-1116.

27. Dutta A, Smith-Jack F, Saunders WP (2014) Prevalence of periradicular periodontitis in a Scottish subpopulation found on CBCT images. International Endodontic Journal 47: 854-863. 
28. Huumonen S, Suominen AL, Vehkalahti MM (2016) Prevalence of apical periodontitis in root filled teeth: findings from a nationwide survey in Finland. International Endodontic Journal 50: 229-226.

29. Loftus JJ, Keating AP, McCartan BE (2005) Periapical status and quality of endodontic treatment in an adult Irish population. International Endodontic Journal 38: 81-86.

30. Segura-Egea JJ, Jiménez-Pinzón A, Poyato-Ferrera M, Velasco-Ortega E, Ríos-Santos JV (2004) Periapical status and quality of root fillings and coronal restorations in an adult Spanish population. International Endodontic Journal 37: $525-530$.

31. Claesson R, Edlund MB, Persson S, Carlsson J (1990) Production of volatile sulfur compounds by various Fusobacterium species. Oral Microbiology and Immunology. Wiley-Blackwell 5:137-142.

32. Carbonero F, Benefiel AC, Alizadeh-Ghamsari AH, Gaskins HR (2012)
Microbial pathways in colonic sulfur metabolism and links with health and disease. Front Physiol 3:448.

33. Attene-Ramos MS, Wagner ED, Plewa MJ (2006) Evidence that hydrogen sulfide is a genotoxic agent. Mol Cancer Res 4: 9-14.

34. Green T, Walton R, Taylor J, Merrell P (1997) RadiographicRadiographic and histological periapical findings of root canal treated teeth in cadaver. Oral Surg Oral Med Oral Pathol Oral Radiol Endod 83:707-711.

35. Piannotti R, Lachette S, Dills S (1986) Desulfuration of cysteine and methionine by Fusobacterium nucleatum J Dent Res 65: 913-917.

36. Nagaoka S, Miyazaki Y, Liu H-J, Iwamoto Y, Kitano M , et al. (1995) Bacteria Invasion into Dentinal Tubules of Human Vital and Nonvital Teeth; Journal of Endodontics 21: 70-73. 